\title{
New Bi-Gravity from New Massive Gravity
}

\author{
A. Akhavan, ${ }^{a}$ M. Alishahiha, ${ }^{b}$ A. Naseh, ${ }^{a}$ A. Nemati ${ }^{c}$ and A. Shirzad ${ }^{c, a}$ \\ ${ }^{a}$ School of Particles and Accelerators, Institute for Research in Fundamental Sciences (IPM), \\ P.O. Box 19395-5531, Tehran, Iran \\ ${ }^{b}$ School of Physics, Institute for Research in Fundamental Sciences (IPM), \\ P.O. Box 19395-5531, Tehran, Iran \\ ${ }^{c}$ Department of Physics, Isfahan University of Technology, \\ P.O. Box 84156-83111, Isfahan, Iran \\ E-mail: amin_akhavan@ipm.ir, alishah@ipm.ir, naseh@ipm.ir, \\ a.nemati@ph.iut.ac.ir, shirzad@ipm.ir
}

ABSTRACT: Using the action of three dimensional New Massive Gravity (NMG) we construct a new bi-gravity in three dimensions. This can be done by promoting the rank two auxiliary field appearing in the expression of NMG's action into a dynamical field. We show that small fluctuations around the AdS vacuum of the model are non-tachyonic and ghost free within certain range of the parameters of the model. We study central charges of the dual field theory and observe that in this range they are positive too. This suggests that the proposed model might be a consistent three dimensional bi-gravity.

KEYwords: Classical Theories of Gravity, Gauge-gravity correspondence, Models of Quantum Gravity

ArXiv ePrint: 1603.03270 


\section{Contents}

1 Introduction 1

2 Solutions of equations of motion 4

2.1 AdS vacuum solution 4

2.2 BTZ black hole solution 5

2.3 Ads wave solutions 5

$\begin{array}{llr}3 & \text { Linearization } & 7\end{array}$

4 Central charges of dual theory $\quad 11$

5 Conclusions $\quad 15$

$\begin{array}{ll}\text { A Details of variation of action } & 16\end{array}$

$\begin{array}{ll}\text { B Details of linearization } & 17\end{array}$

$\begin{array}{ll}\text { C Central charge from entropy function } & 18\end{array}$

$\begin{array}{ll}\mathrm{D} \tilde{\sigma}=-1 \text { case } & 20\end{array}$

\section{Introduction}

Three dimensional Einstein gravity, with a negative cosmological constant, may be thought of as a simplified model to explore and understand quantum gravity [1]. It seems simpler than higher dimensional gravity in the sense that it does not contain local propagating modes, though it is still non-trivial as it has black hole solutions (BTZ solutions) [2] and boundary propagating modes. A natural question or hope is whether one can understand black hole physics in this model. Actually three dimensional gravity, and in particular its BTZ black hole solutions, have increased our knowledge in understanding the AdS/CFT correspondence [3].

To make three dimensional Einstein gravity more realistic, in comparison with higher dimensions, (in the sense of having local propagating modes) one may deform the theory by higher order derivative terms. ${ }^{1}$ In particular one can modify three dimensional Einstein gravity by adding a gravitational Chern-Simon term which leads to a new theory known as Topologically Massive Gravity (TMG) [6]. Another deformation could be done by adding particular curvature squared terms to Einstein gravity leading to a new theory; new Massive

\footnotetext{
${ }^{1}$ For some recent works on massive gravity theories, see $[4,5]$.
} 
Gravity (NMG) [7]. In the latter case one gets two massive propagating gravitons [8]; however, in the former one there is only one degree of freedom, due to chirality.

As a result of presence of higher derivative terms, one expects some inconsistencies in the sense that the model could be tachyonic or could contain ghost. Actually for generic values of parameters of the models, the corresponding equations of motion admit several solutions including AdS, BTZ. It is, however, known that these models suffer from the fact that the energy of graviton and the mass of BTZ black holes cannot be positive at the same time. More precisely, tuning parameters to have positive energy for graviton results to BTZ solutions with negative mass, and vice versa. Equivalently, if one thinks of the model as a theory which provides a gravitational dual for a two dimensional conformal field theory (CFT), the corresponding CFT has negative central charge whenever the propagating spin2 mode in the bulk has positive energy. This problem is known as "bulk-boundary clash".

It was proposed [9] that TMG model could become well defined if one tunes the parameters of the model on particular values (critical points), so that the modes with negative mass are removed. It is, however, observed [10] that at the "critical points" the model admits new "logarithmic" modes which again results to a non-unitray theory (see also $[11,12]$ ). The situation is the same for NMG $[13,14] .^{2}$

In the case of TMG in order to circumvent the above problem, the authors of ref. [18] proposed a new model, named Minimal Massive Gravity (MMG), which has the same minimal local structure as that of TMG. Indeed, working within the "Chern-Simons-like" formulation of massive gravity [19] one may find an action for the MMG model from that of TMG by adding an extra term. Although adding this extra term would open up a possibility to get a consistent ghost free and non-tachyonic three dimensional theory, its linearization about a flat or AdS vacuum still has a single massive mode.

The aim of this paper is to explore a possibility of resolving bulk-boundary clash in the NMG model. To proceed we note that the action of NMG model

$$
I=\frac{1}{16 \pi G} \int d^{3} x \sqrt{-g}\left\{\sigma R-2 \Lambda_{g}-\frac{1}{m^{2}}\left(R^{\mu \nu} R_{\mu \nu}-\frac{3}{8} R^{2}\right)\right\}
$$

may be recast to the following form, using an auxiliary field $f_{\mu \nu}[7]$

$$
I=\frac{1}{16 \pi G} \int d^{3} x \sqrt{-g}\left(\sigma R+f_{\mu \nu} \mathcal{G}^{\mu \nu}+\frac{1}{4} m^{2}\left(\tilde{f}^{\mu \nu} f_{\mu \nu}-\tilde{f}^{2}\right)-2 \Lambda_{g}\right),
$$

where $\sigma= \pm 1$ controls the sign of the curvature term, $G$ is the Newton constant, $m$ is a mass parameter, $\Lambda_{g}$ is a cosmological parameter, $R$ is the Ricci-Scalar and $\mathcal{G}_{\mu \nu}$ is the Einstein tensor of the metric $g_{\mu \nu}$. Here we have used a notation in which $\tilde{f}^{\mu \nu} \equiv g^{\mu \alpha} g^{\nu \beta} f_{\alpha \beta}$, $\tilde{f}_{\nu}^{\mu} \equiv g^{\mu \alpha} f_{\alpha \nu}$ and $\tilde{f} \equiv g^{\mu \nu} f_{\mu \nu}$. Obviously the auxiliary tensor field $f_{\mu \nu}$ is not a dynamical field and can be solved using its algebraic equations of motion. Indeed varying the action with respect to $f_{\mu \nu}$ one finds

$$
f_{\mu \nu}=-\frac{2}{m^{2}}\left(R_{\mu \nu}-\frac{1}{4} g_{\mu \nu} R\right) .
$$

Plugging this expression into the action (1.2) one finds the original action (1.1).

\footnotetext{
${ }^{2}$ For logarithmic solution in higher dimensional gravity see, e.g. [15-17].
} 
The basic idea of our procedure to address the bulk-boundary clash is to promote the auxiliary field $f_{\mu \nu}$ into a dynamical field by adding a new kinetic term for it to the action. Since the resultant model has two dynamical spin-2 fields, this theory can be thought of as a New Bi-Gravity model (NBG). To be precise we will consider the following action ${ }^{3}$

$$
\begin{gathered}
I=\frac{1}{16 \pi G} \int d^{3} x \sqrt{-g}\left(\sigma R[g]+f_{\mu \nu} \mathcal{G}^{\mu \nu}[g]+\frac{1}{4} m^{2}\left(\tilde{f}^{\mu \nu} f_{\mu \nu}-\tilde{f}^{2}\right)-2 \Lambda_{g}\right) \\
+\frac{1}{16 \pi \tilde{G}} \int d^{3} x \sqrt{-f}\left(R[f]-2 \Lambda_{f}\right),
\end{gathered}
$$

where $\Lambda_{f}$ is a new cosmological parameter, $\tilde{G}$ is the Newton constant of the second metric, $R[g]$ and $R[f]$ are Ricci scalars constructed from $g_{\mu \nu}$ and $f_{\mu \nu}$, respectively. $\mathcal{G}_{\mu \nu}[g]$ is also the Einstein tensor of the metric $g_{\mu \nu}$. It is important to note that all indices are raised by $g^{\mu \nu}$ except those in the definition of Ricci scalar $R[f]$ which are raised by the inverse metric $f^{\mu \nu}$.

It is worth noting that there are other bi-gravity models in the literature where the authors have also provided a framework to address the bulk-boundary clash. In particular motivated by the earlier work [20] the authors of [21] introduced a new three dimensional bi-gravity model. Although one can manage to get a positive central charge in this model, the model, in fact, is not unitary in the bulk due to the Boulware-Deser ghost.

Actually our model is very close to another bi-gravity model introduced in [22] to resolve the bulk-boundary clash in NMG. Although the aim of this paper was to address the same question as ours for the NMG model, an advantage of our model is that it can be written using metric formalism, though the one in [22] is formulated using the first order Dreibein formalism. Of course the price we paid is that our model contains non-trivial potential given by the Einstein tensor which makes the model more complicated to study!

To find the equations of motion of the NBG action one should vary the above action with respect to the metrics $g_{\mu \nu}$ and $f_{\mu \nu}$. Doing so one arrives at

$$
\begin{aligned}
\sigma \mathcal{G}[g]_{\mu \nu} & +\Lambda_{g} g_{\mu \nu}=-\frac{m^{2}}{2}\left[\tilde{f}_{\mu}^{\rho} f_{\nu \rho}-\tilde{f} f_{\mu \nu}-\frac{1}{4} g_{\mu \nu}\left(\tilde{f}^{\rho \sigma} f_{\rho \sigma}-\tilde{f}^{2}\right)\right]-2 \tilde{f}_{(\mu}{ }^{\rho} \mathcal{G}[g]_{\nu) \rho} \\
& -\frac{1}{2} f_{\mu \nu} R[g]+\frac{1}{2} \tilde{f} R_{\mu \nu}[g]+\frac{1}{2} g_{\mu \nu} f_{\rho \sigma} \mathcal{G}[g]^{\rho \sigma}-\frac{1}{2}\left[\nabla^{2}[g] f_{\mu \nu}-2 \nabla[g]^{\rho} \nabla[g]_{(\mu} f_{\nu) \rho}\right. \\
& \left.+\nabla[g]_{\mu} \nabla[g]_{\nu} \tilde{f}+\left(\nabla[g]^{\rho} \nabla[g]^{\sigma} f_{\rho \sigma}-\nabla^{2}[g] \tilde{f}\right) g_{\mu \nu}\right] \\
\mathcal{G}_{\mu \nu}[f] & +\Lambda_{f} f_{\mu \nu}=\frac{1}{\kappa} \sqrt{\frac{g}{f}}\left[f_{\alpha \mu} f_{\beta \nu} \mathcal{G}[g]^{\alpha \beta}+\frac{1}{2} m^{2}\left(g^{\sigma \alpha} g^{\tau \beta}-g^{\sigma \tau} g^{\alpha \beta}\right)\left(f_{\sigma \tau} f_{\alpha \mu} f_{\beta \nu}\right)\right]
\end{aligned}
$$

where $\mathcal{G}_{\mu \nu}[f]$ is the Einstein tensor of the metric $f_{\mu \nu}$ and $\kappa=\frac{G}{\tilde{G}}$ is the relative strength of two Newton constants associated with two metrics.

Having proposed a model one should first check whether the model contains non-trivial physics. In order to address this question we will first study different solutions of the above equations of motion. Of course for simplicity we will mainly consider solutions where two

\footnotetext{
${ }^{3}$ Note that we have an option to consider the kinetic term of the second metric with either signs by adding $\tilde{\sigma} R[f]$ with $\tilde{\sigma}= \pm 1$. Of course it what follows we set $\tilde{\sigma}=1$ and study the model in more details. The results of $\tilde{\sigma}=-1$ will be briefly presented in the appendix D.
} 
metrics are proportional. Restricted to this set of solutions, we will see that the model admits several solutions including AdS vacuums, AdS black hole and AdS wave solutions. Then we will study small fluctuations of the metrics around a vacuum solution, where both metrics are AdS whose radius are proportional.

We will see that within a particular range of the parameters the model is consistent in the sense that, at quadratic level, it is ghost free and non-tachyonic. We will also make a comments on the number of degrees of freedom of NBG. Moreover, assuming that the model provides a holographic description for a two dimensional CFT, we will compute central charges of the corresponding CFT and show that within the same range of parameters they are positive as well. Therefore, our model could be a candidate to address a solution to bulk-boundary-clash in NMG.

The paper is organized as follows. In the next section we will present different solutions of the equations of motion (1.5). In section three we will study the linearized equations of motion where we will see that there is a range of parameters over which the model is consistent. ${ }^{4}$ In section four we compute central charges for the dual CFT's. The last section is devoted to discussions.

\section{Solutions of equations of motion}

In this section we shall investigate some possible solutions of the equations of motion (1.5). To be concrete, we will first consider particular solutions in which two metrics $g_{\mu \nu}$ and $f_{\mu \nu}$ are proportional, i.e. $f_{\mu \nu}=\gamma g_{\mu \nu}$. Although this might contains a small subset of general possible solutions of the equations of motion, it is still non-trivial to explore different features of the model. We will also present a non-proportional solutions as well.

\subsection{AdS vacuum solution}

We first investigate the existence of two proportional AdS solution

$$
d s_{g}^{2}=\frac{\ell_{g}^{2}}{r^{2}}\left(d r^{2}-2 d u d v\right), \quad d s_{f}^{2}=\frac{\ell_{f}^{2}}{r^{2}}\left(d r^{2}-2 d u d v\right) .
$$

Substituting the above ansatz in the equations of motion (1.5) one finds

$$
\sigma+\ell_{g}^{2} \Lambda_{g}-\frac{m^{2} \ell_{f}^{4}}{4 \ell_{g}^{2}}+\frac{\ell_{f}^{2}}{2 \ell_{g}^{2}}=0, \quad 1-m^{2} \ell_{f}^{2}-\kappa \frac{\ell_{g}}{\ell_{f}}\left(1+\Lambda_{f} \ell_{f}^{2}\right)=0 .
$$

Assuming $\ell_{f}^{2}=\gamma \ell_{g}^{2}$, the eqs. (2.2) give the following equations to determine $\gamma$ and $\ell_{g}^{2}$

$$
\ell_{g}^{2}=-\frac{\sigma+\frac{1}{2} \gamma}{\Lambda_{g}-\frac{1}{4} m^{2} \gamma^{2}}=\frac{-\kappa+\sqrt{\gamma}}{\gamma\left(m^{2} \sqrt{\gamma}+\kappa \Lambda_{f}\right)}
$$

From the above relations, we find an equation for $\gamma$ as follows

$$
E[\gamma] \equiv a_{5} \gamma^{5}+a_{4} \gamma^{4}+a_{3} \gamma^{3}+a_{2} \gamma^{2}+a_{1} \gamma-\Lambda_{g}^{2}=0
$$

\footnotetext{
${ }^{4}$ We would like to emphasis that by "consistent" we mean that the model at the quadratic level is ghost free and non-tachyonic and central charges of dual theory are positive.
} 
where

$$
\begin{array}{ll}
a_{1}=\Lambda_{g}\left(\frac{\Lambda_{g}}{\kappa^{2}}+2 \sigma \Lambda_{f}\right), & a_{2}=\frac{2 \Lambda_{g} \sigma m^{2}}{\kappa^{2}}-\Lambda_{f}^{2}+\left(\frac{1}{2} m^{2}+\Lambda_{f}\right) \Lambda_{g}, \\
a_{3}=\frac{m^{2}}{\kappa^{2}}\left(m^{2}+\frac{1}{2} \Lambda_{g}\right)-\left(\frac{1}{2} m^{2}+\Lambda_{f}\right) \sigma \Lambda_{f}, & a_{4}=\frac{\sigma m^{4}}{2 \kappa^{2}}-\frac{1}{4}\left(\frac{1}{2} m^{2}+\Lambda_{f}\right)^{2} \\
a_{5}=\frac{m^{4}}{16 \kappa^{2}} . &
\end{array}
$$

Note that the coefficient of $\gamma^{5}$ in eq. (2.4) is positive, thus the function $E[\gamma]$ approaches $\pm \infty$ as one takes the limit of $\gamma \rightarrow \pm \infty$. Moreover one has $E[0]=-\Lambda_{g}^{2}<0$. Therefore, $E[\gamma]$ would certainly intersect with the $\gamma$-axis in (at least) one positive point. This analysis shows that the equations of motion do have proportional AdS solutions.

\subsection{BTZ black hole solution}

Actually it is clear that since the NBG model admits AdS vacuum solutions, it should also has BTZ black holes. This is simply because these black holes are locally AdS. Nonetheless in this subsection we will redo the same computations as we did in the previous subsection for the non-rotating BTZ black hole. Let us consider the following ansatz

$$
d s_{g}^{2}=\ell_{g}^{2}\left[\left(r^{2}-r_{+}^{2}\right) d \tau^{2}+\frac{1}{\left(r^{2}-r_{+}^{2}\right)} d r^{2}+r^{2} d \phi^{2}\right], \quad d s_{f}^{2}=\gamma d s_{g}^{2} .
$$

Plugging this ansatz into the equations of motion (1.5) one arrives at

$$
4 \sigma-\gamma^{2} \ell_{g}^{2} m^{2}+2 \gamma+4 \ell_{g}^{2} \Lambda_{g}=0, \quad 1-m^{2} \ell_{f}^{2}-\kappa \frac{\ell_{g}}{\ell_{f}}\left(1+\Lambda_{f} \ell_{f}^{2}\right)=0
$$

which is indeed the same as those in the eq.(2.3) as it must be. Actually this is the case simply because the solution is locally AdS. Having found the same equations as that in the previous subsection one could then argue that the solution does indeed exist.

\subsection{Ads wave solutions}

So far we have considered solutions in which two metrics are proportional. As an example in this subsection we shall consider a non-proportional solution of the equations of motion. To start, we will consider the following AdS wave ansatz for $g_{\mu \nu}$ and $f_{\mu \nu}$

$$
\begin{aligned}
& d s_{g}^{2}=\frac{\ell_{g}^{2}}{r^{2}}\left(d r^{2}-2 d u d v+G(u, r) d u^{2}\right), \\
& d s_{f}^{2}=\frac{\ell_{f}^{2}}{r^{2}}\left(d r^{2}-2 d u d v+F(u, r) d u^{2}\right) .
\end{aligned}
$$

Note that this ansatz contains two free parameters $\ell_{g}$ and $\ell_{f}$ and two arbitrary functions $G(u, r)$ and $F(u, r)$. Now the aim is to find these parameters and functions using the equations of motion. Indeed plugging the ansatz into the equations of motion of metric 
$g_{\mu \nu}$ one finds an algebraic equation among the parameters of the ansatz and also a partial differential equation for the functions $G$ and $F$. The corresponding equations are

$$
\ell_{g}^{2} \Lambda_{g}-\frac{1}{4} m^{2} \frac{\ell_{f}^{4}}{\ell_{g}^{2}}+\sigma+\frac{1}{2} \frac{\ell_{f}^{2}}{\ell_{g}^{2}}=0 .
$$

and

$$
-\left(\frac{\sigma}{2}+\frac{3}{4} \frac{\ell_{f}^{2}}{\ell_{g}^{2}}\right)\left[\frac{\partial^{2} G}{\partial r^{2}}-\frac{1}{r} \frac{\partial G}{\partial r}\right]+\left(\frac{1}{2} \frac{\ell_{f}^{2}}{\ell_{g}^{2}}\right)\left[\frac{\partial^{2} F}{\partial r^{2}}-\frac{1}{r} \frac{\partial F}{\partial r}\right]-\left(-\frac{1}{2} \frac{\ell_{f}^{4}}{\ell_{g}^{2}} m^{2}+\frac{\ell_{f}^{2}}{\ell_{g}^{2}}\right)\left[\frac{G}{r^{2}}-\frac{F}{r^{2}}\right]=0 .
$$

Similarly from the equations of motion of the metric $f_{\mu \nu}$ one gets

$$
1-m^{2} \ell_{f}^{2}=\kappa \frac{\ell_{g}}{\ell_{f}}\left(1+\Lambda_{f} \ell_{f}^{2}\right)
$$

and

$$
\frac{\partial^{2} G}{\partial r^{2}}-\frac{1}{r} \frac{\partial G}{\partial r}-\kappa \frac{\ell_{g}}{\ell_{f}}\left[\frac{\partial^{2} F}{\partial r^{2}}-\frac{1}{r} \frac{\partial F}{\partial r}\right]+\left(2-m^{2} l_{f}^{2}\right)\left[\frac{G}{r^{2}}-\frac{F}{r^{2}}\right]=0 .
$$

The differential equations (2.10) and (2.12) can be solved by considering solutions of the form of $r^{\alpha}$ for functions $G$ and $F$. In particular assuming $\ell_{f}^{2}=\gamma \ell_{g}^{2}$, we find a forth order algebraic equation for $\alpha$ whose solutions are $\alpha=0,2,1 \pm \sqrt{1-A}$ where $A$ is given by

$$
A=\frac{\sqrt{\gamma}\left(-\gamma \ell_{g}^{2} m^{2}+2\right)(2 \sqrt{\gamma} \kappa-\gamma+2 \sigma)}{\kappa(3 \gamma+2 \sigma)-2 \gamma^{3 / 2}} .
$$

For $\alpha=0$ and $\alpha=2$ we have $F=G$ while for $\alpha=1 \pm \sqrt{1-A}$ one has $F=\beta G$, where

$$
\beta=\frac{A\left(\frac{3}{4} \gamma+\frac{\sigma}{2}\right)-\gamma\left(1-\frac{1}{2} \gamma m^{2} \ell_{g}^{2}\right)}{A\left(\frac{1}{2} \gamma\right)-\gamma\left(1-\frac{1}{2} \gamma m^{2} \ell_{g}^{2}\right)}
$$

Putting everything together one can write the most general solutions for functions $F$ and $G$ as follows

$$
\begin{aligned}
& F(u, r)=f_{1}(u)+f_{2}(u) r^{2}+\beta f_{3}(u) r^{1+\sqrt{1-A}}+\beta f_{4}(u) r^{1-\sqrt{1-A}} \\
& G(u, r)=f_{1}(u)+f_{2}(u) r^{2}+f_{3}(u) r^{1+\sqrt{1-A}}+f_{4}(u) r^{1-\sqrt{1-A}}
\end{aligned}
$$

where $f_{i}(u)$ 's are arbitrary functions of $u$.

It is evident from the above solutions that the equations of motion degenerate at certain points where the model exhibits logarithmic solutions. More precisely these points are given at $A=0$ and $A=1$. In particular for $A=0$, from which ${ }^{5} \sigma-\frac{\gamma}{2}+\kappa \sqrt{\gamma}=0$, one finds

$$
\begin{aligned}
& d s_{g}^{2}=\frac{\ell_{g}^{2}}{r^{2}}\left(d r^{2}-2 d u d v+\left[\tilde{G}_{0}[u] \log (r)+G_{0}[u]+\tilde{G}_{2}[u] r^{2} \log (r)+G_{2}[u] r^{2}\right] d u^{2}\right), \\
& d s_{f}^{2}=\frac{\gamma \ell_{g}^{2}}{r^{2}}\left(d r^{2}-2 d u d v+\left[\tilde{F}_{0}[u] \log (r)+F_{0}[u]+\tilde{F}_{2}[u] r^{2} \log (r)+F_{2}[u] r^{2}\right] d u^{2}\right) .
\end{aligned}
$$

\footnotetext{
${ }^{5}$ Note that setting $A=0$ one gets another solution given by $m^{2} \ell_{g}^{2} \gamma=2$ which, as we will see, should be excluded due to scalar ghost free condition.
} 
It is worth noting that due to the logarithmic behavior of the solution they are not asymptotically AdS solutions. Indeed from AdS/CFT correspondence one could associate two sources with each metric and therefore the corresponding dual theory would be a logarithmic CFT. Having two metrics with logarithmic behavior it would be interesting to explore the physical content of the dual logarithmic CFT.

On the other hand for $A=1$ one finds

$$
\begin{aligned}
& d s_{g}^{2}=\frac{\ell_{g}^{2}}{r^{2}}\left(d r^{2}-2 d u d v+\left[G_{0}[u]+\tilde{G}_{1}[u] r \log (r)+G_{1}[u] r+G_{2}[u] r^{2}\right] d u^{2}\right), \\
& d s_{f}^{2}=\frac{\gamma \ell_{g}^{2}}{r^{2}}\left(d r^{2}-2 d u d v+\left[F_{0}[u]+\tilde{F}_{1}[u] r \log (r)+F_{1}[u] r+F_{2}[u] r^{2}\right] d u^{2}\right) .
\end{aligned}
$$

For this solution one has

$$
m^{2}=\frac{\kappa(\gamma-2 \sigma)+4 \sqrt{\gamma} \sigma}{\gamma^{3 / 2} \ell_{g}^{2}(2 \sigma+2 \sqrt{\gamma} \kappa-\gamma)} .
$$

Note that in this case although the solutions still exhibit logarithmic terms, they are, indeed, asymptotically AdS solutions.

\section{Linearization}

In this section we would like to study small fluctuations of metrics around an AdS vacuum solution. This study may be used to examine the consistency of the NBG model. More precisely one could see whether the propagating modes above this vacuum is tachyonic or the corresponding modes are ghost, or under which conditions the resultant model would be ghost free and non-tachyonic.

To proceed let us consider the following AdS vacuum solution

$$
d s_{g}^{2}=\bar{g}_{\mu \nu} d x^{\mu} d x^{\nu}=\frac{\ell^{2}}{r^{2}}\left(-d t^{2}+d x^{2}+d r^{2}\right), \quad d s_{f}^{2}=\gamma d s_{g}^{2},
$$

and parametrize the fluctuations above this vacuum as follows

$$
g_{\mu \nu}=\bar{g}_{\mu \nu}+h_{\mu \nu}, \quad f_{\mu \nu}=\gamma\left(\bar{g}_{\mu \nu}+\rho_{\mu \nu}\right) .
$$

In order to write the quadratic action with respect to the fluctuations one need to expand various terms of the action up to second order in the metric perturbations $h_{\mu \nu}$ and $\rho_{\mu \nu}$. The details of calculations are given in appendix B. It is clear that the linear part of the action vanishes. On the other hand using the results presented in the appendix $\mathrm{B}$, the quadratic part of the action is found

$$
\begin{aligned}
S^{(2)}\left[h_{\mu \nu}, \rho_{\mu \nu}\right]=\frac{1}{16 \pi G} \int & d^{3} x \sqrt{-\bar{g}}\left\{\left(\sigma+\frac{3}{2} \gamma\right) h^{\mu \nu}\left(\mathbb{G}^{\ell} h\right)_{\mu \nu}+\kappa \sqrt{\gamma} \rho^{\mu \nu}\left(\mathbb{G}^{\ell} \rho\right)_{\mu \nu}\right. \\
& \left.-2 \gamma h^{\mu \nu}\left(\mathbb{G}^{\ell} \rho\right)_{\mu \nu}+(h-\rho) \cdot(h-\rho)\right\},
\end{aligned}
$$

where $\mathbb{G}^{\ell}$ is the Pauli-Fierz operator on the curved $A d S_{3}$ background with radius $\ell$. For two arbitrary second rank tensors $p$ and $q$ the Pauli-Fierz operator is

$$
p^{\mu \nu}\left(\mathbb{G}^{\ell} q\right)_{\mu \nu} \equiv-\frac{1}{4} p_{\nu \rho ; \mu} q^{\nu \rho ; \mu}+\frac{1}{2} p_{\mu \nu ; \rho} q^{\rho \nu ; \mu}-\frac{1}{4} p_{; \mu} q_{; \nu}^{\mu \nu}-\frac{1}{4} q_{; \mu} p_{; \nu}^{\mu \nu}+\frac{1}{4} p_{; \mu} q^{; \mu}
$$




$$
-\frac{1}{\ell^{2}}\left(p^{\mu \nu} q_{\mu \nu}-\frac{1}{2} p q\right)
$$

where "; " denotes covariant derivative with respect to the background metric. The inner product used in eq. (3.3) is also defined by

$$
h \cdot \rho \equiv \chi h_{\mu \nu} \rho^{\mu \nu}-\xi h \rho,
$$

where $h=\bar{g}^{\mu \nu} h_{\mu \nu}, \rho=\bar{g}^{\mu \nu} \rho_{\mu \nu}, \rho^{\mu \nu}=\bar{g}^{\mu \mu^{\prime}} \bar{g}^{\nu \nu^{\prime}} \rho_{\mu^{\prime} \nu^{\prime}}$ and

$$
\chi=-\frac{1}{4} m^{2} \gamma^{2}+\frac{1}{2} \frac{\gamma}{\ell^{2}}, \quad \xi=\frac{1}{4} \frac{\gamma}{\ell^{2}} .
$$

To investigate whether the model is ghost free, it is useful to utilize a new basis for the fluctuations

$$
\rho=a h^{(m)}+h^{(0)}, \quad h=h^{(m)}+b h^{(0)},
$$

by which the quadratic part of the action in terms of $h^{(0)}$ and $h^{(m)}$ reads

$$
\begin{array}{rl}
S^{(2)}\left[h^{(0)}, h^{(m)}\right]=\frac{1}{16 \pi G} \int d^{3} & x \sqrt{-\bar{g}}\left[\left(\left(\sigma+\frac{3}{2} \gamma\right) b^{2}+\kappa \sqrt{\gamma}-2 \gamma b\right) h^{(0) \mu \nu}\left(\mathbb{G}^{\ell} h^{(0)}\right)_{\mu \nu}\right. \\
+ & \left(\sigma+\frac{3}{2} \gamma+\kappa \sqrt{\gamma} a^{2}-2 \gamma a\right) h^{(m) \mu \nu}\left(\mathbb{G}^{\ell} h^{(m)}\right)_{\mu \nu} \\
+ & 2\left(\left(\sigma+\frac{3}{2} \gamma\right) b+\kappa \sqrt{\gamma} a-\gamma(1+a b)\right) h^{(0) \mu \nu}\left(\mathbb{G}^{\ell} h^{(m)}\right)_{\mu \nu} \\
+ & (b-1)^{2} \chi\left(h^{(0) \mu \nu} h_{\mu \nu}^{(0)}-\frac{\xi}{\chi}\left(h^{(0)}\right)^{2}\right) \\
+ & (a-1)^{2} \chi\left(h^{(m) \mu \nu} h_{\mu \nu}^{(m)}-\frac{\xi}{\chi}\left(h^{(m)}\right)^{2}\right) \\
& \left.-2(b-1)(a-1) \chi\left(h^{(0) \mu \nu} h_{\mu \nu}^{(m)}-\frac{\xi}{\chi} h^{(0)} h^{(m)}\right)\right] .
\end{array}
$$

The procedure is to fix the parameters $a$ and $b$ in such a way that the above action reduces to two decoupled actions for modes $h^{(0)}$ and $h^{(m)}$. This can be done by setting the coefficients of the cross terms (in the third and sixth lines of eq. (3.8)) to zero

$$
\left(\sigma+\frac{3}{2} \gamma\right) b+\kappa \sqrt{\gamma} a-\gamma(1+a b)=0, \quad(b-1)(a-1)=0 .
$$

Moreover to make sure that the resultant action does not have scalar ghost one should further set $\chi=\xi[23,24]$ leading to $m^{2} \ell^{2} \gamma=1$. Now the aim is to see whether we could find a range for parameters of the above action over which it is ghost free and non-tachyonic.

To proceed we note that the solution $a=b=1$ should be discarded. This is because for this solution the condition (3.9) reduces to $\sigma-\gamma / 2+\sqrt{\gamma} \kappa=0$ which is exactly the condition where the NBG model exhibits a logarithmic solution. This fact is also clear from the action (3.8) as at this point the modes $h^{(0)}$ and $h^{(m)}$ degenerate resulting to two massless modes. Therefore in what follows we should restrict ourselves to the cases of $b=1, a \neq 1$ or $a=1, b \neq 1$. It is, however, easy to see that these two cases are essentially 
equivalent and thus it is enough to consider only one of them. In what follows we will consider the first case in which $b=1$. In this case from the equation (3.9) one finds

$$
a=-\frac{m^{2} \ell^{2} \sigma+\frac{1}{2}}{\kappa m \ell-1}
$$

In this case the action (3.8) reads

$$
\begin{aligned}
S^{(2)}\left[h^{(0)}, h^{(m)}\right] & =\frac{1}{16 \pi G} \int d^{3} x \sqrt{-\bar{g}}\left[\mathbb{A}_{0} h^{(0) \mu \nu}\left(\mathbb{G}^{\ell} h^{(0)}\right)_{\mu \nu}+\right. \\
+ & \left.\mathbb{A}_{m}\left\{h^{(m) \mu \nu}\left(\mathbb{G}^{\ell} h^{(m)}\right)_{\mu \nu}-\frac{\mathbb{M}^{2}}{4}\left(h^{(m) \mu \nu} h_{\mu \nu}^{(m)}-\left(h^{(m)}\right)^{2}\right)\right\}\right],
\end{aligned}
$$

where

$$
\begin{aligned}
\mathbb{A}_{0} & =\sigma-\frac{1}{2 m^{2} \ell^{2}}+\frac{\kappa}{m \ell} \\
\mathbb{A}_{m} & =\sigma+\left(\frac{3}{2}-2 a\right) \frac{1}{m^{2} \ell^{2}}+\frac{a^{2} \kappa}{m \ell} \\
\mathbb{M}^{2} & =-\frac{1}{\ell^{2}} \frac{(a-1)^{2}}{m^{2} \ell^{2} \mathbb{A}_{m}}
\end{aligned}
$$

In order to get a ghost free and non-tachyonic model certain conditions should be imposed on the parameters of the action. More precisely from ghost free condition one has

$$
\mathbb{A}_{0}>0, \quad \mathbb{A}_{m}>0
$$

while the model is non-tachyonic if ${ }^{6}$

$$
\mathbb{M}^{2} \geq-\frac{1}{\ell^{2}}
$$

One can investigate allowed values for $\left(m^{2} \ell^{2}, \kappa\right)$ so that both ghost free and non-tachyonic conditions are satisfied. The allowed values of $m^{2} \ell^{2}$ and $\kappa$ for the cases of $\sigma=1$ and $\sigma=-1$ are shown in figure 1. For these values of $\left(m^{2} \ell^{2}, \kappa\right)$ the massive modes acquires different masses in the range $-\frac{1}{\ell^{2}} \leq \mathbb{M}^{2}<0$ as depicted in figure 2 .

For generic value of $\mathbb{M}^{2} \neq-\frac{1}{\ell^{2}}$ the spectrum of the NBG model consists of a massless graviton mode and a massive spin-2 field which has two degrees of freedom. On the other hand when the bound saturates at $\mathbb{M}^{2}=-\frac{1}{\ell^{2}}$ one gets a partially massive mode which, unlike massive spin-2 field, has just one degree of freedom at the linear level. Actually it has been shown that the Pauli-Fierz action for this special value of mass has an extra gauge symmetry which affects the number of degrees of freedom [29-33]. ${ }^{7}$ We note, however, that

\footnotetext{
${ }^{6}$ It is argued that for an $\mathrm{AdS}_{3}$ vacuum with radius $\ell$, the unitarity allows the massive spin- 2 to have a negative mass squared $\mathbb{M}^{2}$ provided $\mathbb{M}^{2} \geq-1 / \ell^{2}[25-30]$.

${ }^{7}$ Indeed the Pauli-Fierz action for this special value of mass has an gauge symmetry generated by a scalar gauge parameter $\chi(x)$ as follows $[29,30]$

$$
\delta_{\chi} h_{\mu \nu}^{(m)}=\bar{\nabla}_{\mu} \bar{\nabla}_{\nu} \chi-\frac{1}{\ell^{2}} \bar{g}_{\mu \nu} \chi
$$
}



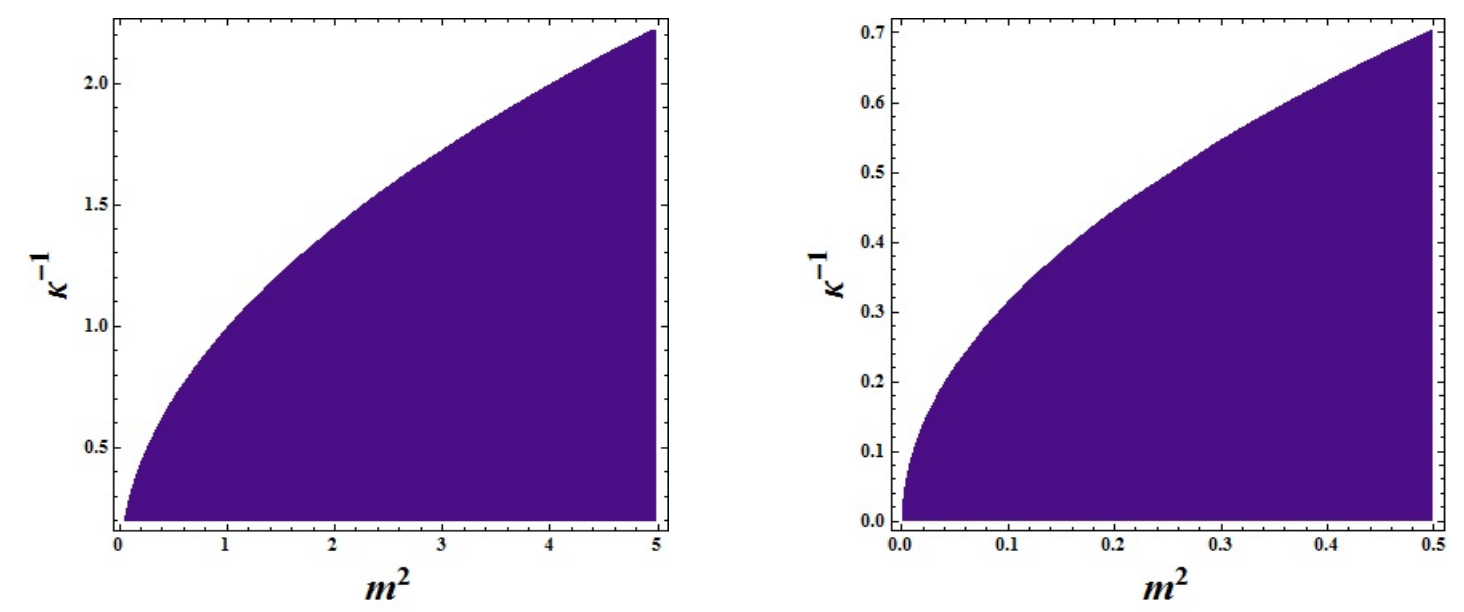

Figure 1. Unitary Region for $m^{2}$ and $\kappa^{-1}$ for the cases $\sigma=1$ (left) and $\sigma=-1$ (right). Note that here we set $\ell=1$.
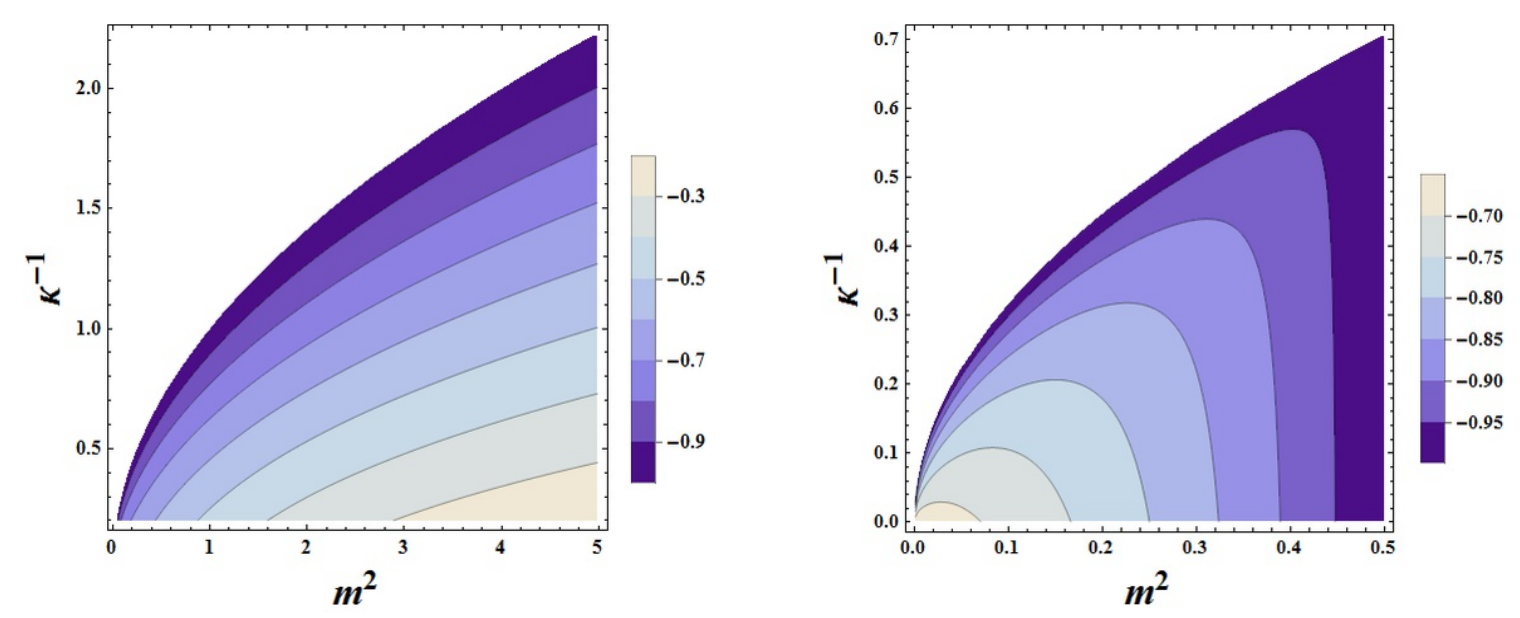

Figure 2. Values of $\mathbb{M}^{2}$ within the unitary region for $m^{2}$ and $\kappa^{-1}$ for the cases $\sigma=1$ (left) and $\sigma=-1$ (right). Note that here we set $\ell=1$.

this gauge symmetry in most theories is an artifact of linear approximation and disappears at the non-linear level $[34,35]$. Of course if the theory is Weyl invariant, such as 3D conformal gravity, this extra gauge symmetry remains in fully non-linear level [36].

In our case the inequality could saturate for $(a=0, b=1)$ in which the action (3.8) reads $^{8}$

$$
\begin{aligned}
S^{(2)}\left[h^{(0)}, h^{(m)}\right] & =\frac{1}{16 \pi G} \int d^{3} x \sqrt{-\bar{g}}\left[(-2+\kappa \sqrt{2}) h^{(0) \mu \nu}\left(\mathbb{G}^{\ell} h^{(0)}\right)_{\mu \nu}\right. \\
+2 & \left.\left\{h^{(m) \mu \nu}\left(\mathbb{G}^{\ell} h^{(m)}\right)_{\mu \nu}+\frac{1}{4 \ell^{2}}\left(h^{(m) \mu \nu} h_{\mu \nu}^{(m)}-\left(h^{(m)}\right)^{2}\right)\right\}\right] .
\end{aligned}
$$

\footnotetext{
${ }^{8}$ Note that partial massive gravity could also appear at $(a=1, b=0)$ where we get $\kappa=\sqrt{\gamma}$. Although this case may be happen for either signs of $\sigma$, the ghost free condition will still be $\kappa>\sqrt{2}$.
} 
Note that this may happen when $\sigma=-1$ and the ghost free condition reduces to $\kappa>\sqrt{2}$. Therefore at this particular value the NBG model has a partially massive mode at the linear level. We note, however, that since the action (1.4) is not Weyl invariant (even for the ghost-free and non-tachyonic parameters), the existence of extra gauge symmetry may be an artifact of linear approximation. It would be interesting to explore this point better along the study of $[34,37,38]$.

\section{Central charges of dual theory}

In this section in order to further investigate the consistency of the NBG model we shall study its properties using AdS/CFT correspondence. More precisely the model, given by the action (1.4), could provide a holographic description for a dual field theory. Indeed if one considers the model on an asymptotically locally AdS solution, one would expect that the dual theory is a CFT theory, though since the gravitational theory has two asymptotically AdS metrics, it is not clear to us what exactly the corresponding CFT could be!

Nonetheless, using the free energy of an asymptotically AdS solution one may compute the entropy of the corresponding solution from which one can read central charges of the dual theory using Cardy formula for entropy [39, 40]. Doing so, one could investigate conditions on which the obtained central charges are positive. Intuitively, one would expect that for a consistent theory the central charges are positive exactly in the range of parameters where the model is non-tachyonic and ghost free.

Note that in general on-shell action is divergent and one needs to regularize it by adding certain boundary terms to the action. These boundary terms are those which are needed to have a well defined variational principle, and those needed to remove infinities in the on-shell action. Of course in general finding these boundary terms is not an easy task, though in what follows we will find them for a subset of solutions where two metrics are proportional.

To proceed we note that the variation of the action (1.4) with respect to $g_{\mu \nu}$ and $f_{\mu \nu}$, in general, contains boundary terms which may invalidate the variational principle for Dirichlet boundary condition. These boundary terms emerge from the following terms

$$
\delta I_{\delta \partial_{r} g, \delta \partial_{r} f}=\frac{1}{16 \pi G} \int d^{3} x\left(\sigma \sqrt{-g} \delta R[g]+\sqrt{-g} f_{\mu \nu} \delta \mathcal{G}^{\mu \nu}[g]+\kappa \sqrt{-f} \delta R[f]\right),
$$

which can be written in the following form

$$
\delta I_{\delta \partial_{r} g, \delta \partial_{r} f}=\frac{1}{16 \pi G} \int d^{3} x\left(\sqrt{-g} \mathcal{A}_{\mu \nu} \delta R^{\mu \nu}[g]+\kappa \sqrt{-f} f_{\mu \nu} \delta R^{\mu \nu}[f]\right)
$$

where

$$
\mathcal{A}_{\mu \nu}=\sigma g_{\mu \nu}+f_{\mu \nu}-\frac{1}{2} f_{\alpha \beta} g^{\alpha \beta} g_{\mu \nu}
$$

From the first term in eq. (4.2) one gets two different boundary terms as follows

$$
\sqrt{-g} \nabla[g]_{\nu}\left(\mathcal{A}^{\mu \nu} \delta \Gamma_{\mu \sigma}^{\sigma}-\mathcal{A}^{\mu \alpha} \delta \Gamma_{\mu \alpha}^{\nu}\right)
$$

and

$$
-\frac{1}{2} \sqrt{-g}\left[\nabla[g]_{\mu}\left(\nabla[g]_{\nu} \mathcal{A}^{\mu \nu} g^{\sigma \rho} \delta g_{\sigma \rho}\right)+\nabla[g]_{\sigma}\left(\nabla[g]_{\nu} \mathcal{A}^{\mu \nu} g^{\sigma \rho} \delta g_{\mu \rho}\right)\right.
$$




$$
\begin{aligned}
& -\nabla[g]_{\rho}\left(\nabla[g]_{\nu} \mathcal{A}^{\mu \nu} g^{\sigma \rho} \delta g_{\mu \sigma}\right)-\nabla[g]_{\mu}\left(\nabla[g]_{\sigma} \mathcal{A}^{\mu \nu} g^{\sigma \rho} \delta g_{\nu \rho}\right)-\nabla[g]_{\nu}\left(\nabla[g]_{\sigma} \mathcal{A}^{\mu \nu} g^{\sigma \rho} \delta g_{\mu \rho}\right) \\
& \left.+\nabla[g]_{\rho}\left(\nabla[g]_{\sigma} \mathcal{A}^{\mu \nu} g^{\sigma \rho} \delta g_{\mu \nu}\right)\right] .
\end{aligned}
$$

Similarly the same form of boundary terms emerge from the variation of action with respect to $f_{\mu \nu}$.

Obviously the boundary terms given in eq. (4.4) (also that for $f_{\mu \nu}$ ) invalidate the variational principle for the Dirichlet boundary condition. Of course these terms could be removed by adding proper Gibbons-Hawking terms. Actually since we are interested in solutions where two metrics are proportional, $f_{\mu \nu}=\gamma g_{\mu \nu}$, the variation of the action may be recast into the following illustrative form

$$
\delta I_{\delta \partial_{r} g, \delta \partial_{r} f}=\frac{1}{16 \pi G} \int d^{3} x\left(\sqrt{-g}\left(\sigma-\frac{\gamma}{2}\right) g_{\mu \nu} \delta R^{\mu \nu}[g]+\kappa \sqrt{-f} f_{\mu \nu} \delta R^{\mu \nu}[f]\right),
$$

from which the proper Gibbons-Hawking terms can be suggested as follows

$$
I_{G H}=-\frac{2\left(\sigma-\frac{\gamma}{2}\right)}{16 \pi G} \int d^{2} x \sqrt{-\eta_{g}} \eta_{g}^{i j} K[g]_{i j}-\frac{2}{16 \pi \tilde{G}} \int d^{2} x \sqrt{-\eta_{f}} \eta_{f}^{i j} K[f]_{i j},
$$

where $\eta_{g_{i j}}$ and $\eta_{f_{i j}}$ are the induced metrics, on the boundary, associated with the metrics $g$ and $f$, respectively. We would like to re-emphasize that the above boundary terms are obtained for a subclass solutions where two metrics are proportional. Nonetheless it is worth comparing these terms with the generalized Gibbons-Hawking term which is obtained in [41] to make the variational principle well defined for the NMG model. Actually one can see that assuming $f_{i j}=\gamma g_{i j}$, the generalized Gibbons-Hawking terms (given in the eq. (2.17) of [41]) exactly reduce to the above boundary term $I_{G H}$ in the limit of $G \rightarrow 0$.

Let us now evaluate the on-shell action consisting of the original bulk action (1.4) (named $I_{0}$ ) and the above Gibbons-Hawking terms for the following BTZ black hole solution

$$
d s_{g}^{2}=\ell^{2}\left[\left(r^{2}-r_{+}^{2}\right) d \tau^{2}+\frac{1}{\left(r^{2}-r_{+}^{2}\right)} d r^{2}+r^{2} d \phi^{2}\right], \quad d s_{f}^{2}=\gamma d s_{g}^{2},
$$

where $r_{+}$is the radius of horizon. Plugging the metrics (4.8) into the action and performing integrations over $\tau, r$ and $\phi$ one arrives at

$$
I_{0}+I_{G H}=\frac{1}{16 \pi G}\left[\left(a_{\mathrm{div}}+\kappa b_{\mathrm{div}}\right) \mathcal{R}^{2}+\left(a_{\mathrm{fin}}+\kappa b_{\mathrm{fin}}\right)\right]
$$

where $\mathcal{R} \gg r_{+}$is a cutoff and

$$
\begin{aligned}
a_{\mathrm{div}} & =(2 \pi)^{2} \frac{\ell}{r_{+}}\left(\sigma-\frac{3}{4} m^{2} \ell^{2} \gamma^{2}-\Lambda_{g} \ell^{2}-\frac{1}{2} \gamma\right), \\
b_{\mathrm{div}} & =(2 \pi)^{2} \frac{\ell}{r_{+}} \sqrt{\gamma}\left(1-\Lambda_{f} \gamma \ell^{2}\right), \\
a_{\mathrm{fin}} & =(2 \pi)^{2} \ell r_{+}\left(\sigma+\frac{3}{4} m^{2} \ell^{2} \gamma^{2}+\Lambda_{g} \ell^{2}-\frac{1}{2} \gamma\right), \\
b_{\mathrm{fin}} & =(2 \pi)^{2} \ell r_{+} \sqrt{\gamma}\left(1+\Lambda_{f} \gamma \ell^{2}\right) .
\end{aligned}
$$


It is clear that the on-shell action is divergent due to the infinite volume limit and proper counterterms are needed to remove the divergent terms. It is easy to see that the corresponding boundary counterterms are

$$
I_{c . t .}=a_{1} \int d^{2} x \sqrt{\eta_{g}}+a_{2} \int d^{2} x \sqrt{\eta_{f}}
$$

where

$$
a_{1}=-\frac{1}{16 \pi G \ell}\left(\sigma-\frac{3}{4} m^{2} \ell^{2} \gamma^{2}-\Lambda_{g} \ell^{2}-\frac{\gamma}{2}\right), \quad a_{2}=-\frac{1}{16 \pi \tilde{G} \ell \sqrt{\gamma}}\left(1-\Lambda_{f} \gamma \ell^{2}\right) .
$$

Putting every things together the renormalized on-shell action reads

$$
I_{\mathrm{ren}}=I_{0}+I_{G H}+I_{c t}=\frac{1}{16 \pi G}\left(\tilde{a}_{f i n}+\kappa \tilde{b}_{f i n}\right)
$$

with

$$
\begin{aligned}
& \tilde{a}_{\text {fin }}=6 \pi^{2} \ell r_{+}\left(\sigma+\frac{1}{4} m^{2} \ell^{2} \gamma^{2}+\frac{1}{3} \Lambda_{g} \ell^{2}-\frac{1}{2} \gamma\right), \\
& \tilde{b}_{\text {fin }}=6 \pi^{2} \ell r_{+} \sqrt{\gamma}\left(1+\frac{1}{3} \Lambda_{f} \gamma \ell^{2}\right) .
\end{aligned}
$$

Using the fact that the free energy is given by $F=-T I_{r e n}$ with $T=\frac{r_{+}}{2 \pi \ell}$ being the Hawking temperature, the entropy may be found as follows

$$
\begin{aligned}
S & =-\frac{\partial F}{\partial T} \\
& =\frac{12 \pi^{2} \ell r_{+}}{16 \pi G}\left[\left(\sigma+\frac{1}{4} m^{2} \ell^{2} \gamma^{2}+\frac{1}{3} \Lambda_{g} \ell^{2}-\frac{1}{2} \gamma\right)+\kappa \sqrt{\gamma}\left(1+\frac{1}{3} \Lambda_{f} \gamma \ell^{2}\right)\right] .
\end{aligned}
$$

To read the central charges one should compare this entropy with the Cardy formula for entropy given by [39]

$$
S=\frac{\pi^{2} \ell}{3}\left(C_{R} T_{R}+C_{L} T_{L}\right)
$$

where $C_{L(R)}$ and $T_{L(R)}$ are central charge and temperature of left (right) moving sector respectively. On the other hand since the model under consideration is parity invariant one has

$$
C_{R}=C_{L} \equiv C, \quad T_{R}=T_{L}=\frac{r_{+}}{2 \pi \ell} .
$$

Therefore comparing eq. (4.15) with (4.16) and using eq. (4.17) one arrives at

$$
C=\frac{1}{16 \pi G}\left(C_{k}+\kappa C_{\tilde{k}}\right)
$$

where

$$
C_{k}=36 \pi \ell\left(\sigma+\frac{1}{4} m^{2} \ell^{2} \gamma^{2}+\frac{1}{3} \Lambda_{g} \ell^{2}-\frac{1}{2} \gamma\right), \quad C_{\tilde{k}}=36 \pi \ell \sqrt{\gamma}\left(1+\frac{1}{3} \Lambda_{f} \gamma \ell^{2}\right) .
$$


It is important to note that since the metrics given in the eq. (4.8) are solutions of the equations of motion, their parameters should be fixed from eq. (2.7). Actually from the equation (2.7) and taking into account the scalar ghost free condition ${ }^{9}$ one finds

$$
\Lambda_{g}=-\frac{1}{\ell^{2}}\left(\sigma+\frac{1}{4 m^{2} \ell^{2}}\right), \quad \Lambda_{f}=-m^{2}, \quad \gamma=\frac{1}{m^{2} \ell^{2}} .
$$

Plugging these expressions into the equation (4.19) one arrives at

$$
C=\frac{3 \ell}{2 G}\left(\sigma-\frac{1}{2 m^{2} \ell^{2}}+\frac{\kappa}{m \ell}\right)
$$

which is the central charge for the CFT dual of the NBG model. Obviously for $\kappa^{2} \rightarrow 0$ where the model reduces to NMG one gets

$$
C=\frac{3 \ell}{2 G}\left(\sigma-\frac{1}{2 m^{2} \ell^{2}}\right)
$$

which is the well-known central charge of the CFT dual to the NMG model on an asymptotically locally $A d S_{3}$ geometry $[28,42]$. Since the way we have obtained the above expression for central charge crucially depends on the boundary terms and also due to its important role to address the bulk-boundary clash, it would be interesting if one could find this expression from another consideration. Indeed in the appendix $\mathrm{C}$ we have re-derived this expression using entropy function formalism by which one may compute the entropy of an extremal black hole [43].

Interestingly enough using the equation (3.12) the above central charge may be recast into the following form

$$
C=\frac{3 \ell}{2 G} \mathbb{A}_{0}
$$

where $\mathbb{A}_{0}$ is the coefficient of the kinetic term of the massless graviton which is positive within the range of our interest (see figure 1). Therefore the absence of scalar ghost and positivity of kinetic terms, trivially implies that the central charge is positive. Note that in this range $\mathbb{A}_{m}$ is also positive. Thus the NBG model could resolve the bulk-boundary clash of the NMG model.

It is worth noting that the central charge may be also recast into the form of $C=$ $\frac{3 \ell}{2 G}\left(\sigma-\frac{\gamma}{2}+\kappa \sqrt{\gamma}\right)$ from which it is clear that setting $C=0$ one arrives at a logarithmic CFT (see also eq. (2.16)).

The relation between central charge and the coefficient of the kinetic term of massless graviton (equation (4.23)) could be simply understood from the fact that the massless graviton is the source for the dual stress-energy tensor. Note also that there is no a cross term between massless graviton and spin-2 field and therefore two-point functions of stressenergy tensor can be entirely obtained from the action of massless graviton. On the other hand, since the corresponding two-point functions are proportional to the dual central

\footnotetext{
${ }^{9}$ Note that since BTZ solution is locally AdS, the scalar ghost free condition in the present case is the same as that of AdS solution; i.e. $\gamma m^{2} \ell^{2}=1$.
} 
charge, the positivity of central charge should be encoded into the positivity of the kinetic term in the action of massless graviton.

Therefore if, for a model, one could provide a condition that both $\mathbb{A}_{0}$ and $\mathbb{A}_{m}$ are positive, this would automatically give positive central charge and resolve bulk-boundary clash too. This is, indeed, the case for the NBG model.

\section{Conclusions}

In this paper we have addressed the bulk-boundary clash for NMG model by proposing a new bi-gravity in three dimensions. To introduce our model we have inspired by NMG action written in terms of a rank two auxiliary field. The idea was to promote the auxiliary field to a dynamical field.

We have shown that the resultant model given by the action (1.4) has non-trivial content in the sense that its equations of motion admit several non-trivial solutions such as BTZ black hole and AdS waves. Of course we have mainly considered a small subset of all possible solutions where two metrics are proportional. In particular we have considered a vacuum solution where both metrics are AdS geometers whose radii are proportional, i.e. $\ell_{f}^{2}=\gamma \ell_{g}^{2}$.

We have also studied small fluctuations around this AdS vacuum where we have shown that at linear level the model consists of a massless graviton and a massive spin- 2 field. We have shown that there is a range for parameters where the NBG model becomes ghost free non-tachyonic and therefore could be classically consistent. It is important to note that for these values of parameters the model has the same degrees of freedom as that of NMG.

We note, however, that for a particular value of parameters the spin- 2 field becomes partially massive which means that in the linearized level it has just one degree of freedom. Of course we would expect that this reduction of degrees of freedom is an artifact of the linear approximation. Accepting this fact we would like to conclude that the NBG model has the same degrees of freedom as that of NMG and therefore could be thought of as a model which addresses solution of bulk-boundary clash for NMG.

Intuitively it is simple to see how our model could resolve bulk-boundary clash. Indeed we have shown that upon linearization the quadratic part of the action contains two parts: one for a massless graviton and one for a massive spin-2 field. Denoting the coefficients of these terms by $\mathbb{A}_{0}$ and $\mathbb{A}_{m}$, respectively, one can see that for NMG model these two factors cannot be positive. Moreover since $\mathbb{A}_{0}$ is related to the central charge of the dual theory one generally gets the bulk-boundary clash. Whereas for the NBG model there is a range of parameters where both $\mathbb{A}_{0}$ and $\mathbb{A}_{m}$ can be positive simultaneously. This range of parameters are given in figure 1.

Of course it is important to note that our study crucially depends on the fact that we have considered a vacuum AdS solution where two metrics are proportional. The theory could have other solutions and linearization around them would be different from that considered in this paper. It will be interesting to study these solutions and fluctuations around them. It is also worth noting that in this paper we have only studied the consistency of the model up to quadratic terms of the action. To fully address the problem one should also consider higher order terms to make sure that the theory remains ghost free. Alternatively 
one may study the constraint system of the full theory using Hamintonian formalism. We hope to address this question in our future work.

Finally we note that although the way we have found the central charge of the dual theory relies on the particular solution we have taken, we think that the expression given in the equation (4.21) is general and could be found by another method such as asymptotic symmetry analysis of the model.

\section{Acknowledgments}

We would like to thank H. Afshar, E. Altas, A. F. Astaneh, E. Bergshoeff, S. Carlip, D. Grumiller, A. Mollabashi, M. R. Mohammdai Mozaffar, F. Omidi, S. F. Taghavi and M. R. Tanhayi for useful discussions. A. N. would also like to thank ICTP where the project is finalized for warm hospitality. We would also like to thank the referee for his/her comments.

\section{A Details of variation of action}

In this appendix we present details of the variation of the action (1.4). For variation with respect to $g^{\mu \nu}$ of various terms we have

$$
\begin{aligned}
\delta \sqrt{-g} & =\frac{-1}{2} \sqrt{-g} g_{\mu \nu} \delta g^{\mu \nu}, \\
\delta(\sqrt{-g} R[g]) & =\sqrt{-g} G_{\mu \nu}[g] \delta g^{\mu \nu}+\mathcal{B}_{1}, \\
\delta\left(\tilde{f}^{\mu \nu} G_{\mu \nu}\right) & =\delta g^{\mu \nu}\left(2 \tilde{f}_{(\mu}{ }^{\rho} G_{\nu) \rho}+\frac{1}{2}\left[\nabla^{2}[g] f_{\mu \nu}-2 \nabla[g]^{\rho} \nabla[g]_{(\mu} f_{\nu) \rho}+\nabla[g]_{\mu} \nabla[g]_{\nu} \tilde{f}\right.\right. \\
& \left.\left.+\left(\nabla[g]^{\rho} \nabla[g]^{\sigma} f_{\rho \sigma}-\nabla^{2}[g] \tilde{f}\right) g_{\mu \nu}\right]+\frac{1}{2} R f_{\mu \nu}-\frac{1}{2} \tilde{f} R_{\mu \nu}\right)+\mathcal{B}_{2}, \\
\delta\left(\tilde{f}^{\mu \nu} f_{\mu \nu}-\tilde{f}^{2}\right) & =2\left(f_{\nu \rho} \tilde{f}_{\mu}^{\rho}-\tilde{f} f_{\mu \nu}\right) \delta g^{\mu \nu},
\end{aligned}
$$

where the boundary term $\mathcal{B}_{1}$ is

$$
\mathcal{B}_{1}=\sqrt{-g} \nabla[g]_{\nu}\left(g^{\mu \nu} \delta \Gamma_{\mu \sigma}^{\sigma}[g]-g^{\mu \alpha} \delta \Gamma_{\mu \alpha}^{\nu}[g]\right),
$$

and the boundary term $\mathcal{B}_{2}$, setting $B_{\mu \nu}=f_{\mu \nu}-\frac{1}{2} f g_{\mu \nu}$, is

$$
\begin{aligned}
\mathcal{B}_{2}= & \sqrt{-g} \nabla[g]_{\nu}\left(B^{\mu \nu} \delta \Gamma_{\mu \sigma}^{\sigma}-B^{\mu \alpha} \delta \Gamma_{\mu \alpha}^{\nu}\right)-\frac{1}{2} \sqrt{-g}\left(\nabla[g]_{\mu}\left(\nabla[g]_{\nu} B^{\mu \nu} g^{\sigma \rho} \delta g_{\sigma \rho}\right)\right. \\
& +\nabla[g]_{\sigma}\left(\nabla[g]_{\nu} B^{\mu \nu} g^{\sigma \rho} \delta g_{\mu \rho}\right)-\nabla[g]_{\rho}\left(\nabla[g]_{\nu} B^{\mu \nu} g^{\sigma \rho} \delta g_{\mu \sigma}\right)-\nabla[g]_{\mu}\left(\nabla[g]_{\sigma} B^{\mu \nu} g^{\sigma \rho} \delta g_{\nu \rho}\right) \\
& \left.-\nabla[g]_{\nu}\left(\nabla[g]_{\sigma} B^{\mu \nu} g^{\sigma \rho} \delta g_{\mu \rho}\right)+\nabla[g]_{\rho}\left(\nabla[g]_{\sigma} B^{\mu \nu} g^{\sigma \rho} \delta g_{\mu \nu}\right)\right) .
\end{aligned}
$$

Variations of different terms in the action (1.4) with respect to $f^{\mu \nu}$ are

$$
\begin{gathered}
\delta \sqrt{-f}=-\frac{1}{2} \sqrt{-f} f_{\mu \nu} \delta f^{\mu \nu}, \\
\delta(\sqrt{-f} R[f])=\sqrt{-f} G_{\mu \nu}[f] \delta f^{\mu \nu}+\mathcal{B}_{3},
\end{gathered}
$$




$$
\begin{aligned}
\delta\left(f_{\mu \nu} G^{\mu \nu}[g]\right) & =-f_{\mu \alpha} f_{\nu \beta} G^{\mu \nu}[g] \delta f^{\alpha \beta} \\
\delta\left(\tilde{f}^{\mu \nu} f_{\mu \nu}-\tilde{f}^{2}\right) & =-2\left(g^{\mu \alpha} g^{\nu \beta}-g^{\mu \nu} g^{\alpha \beta}\right) f_{\alpha \beta} f_{\mu \alpha} f_{\nu \beta} \delta f^{\alpha \beta},
\end{aligned}
$$

where the boundary term $\mathcal{B}_{3}$ is

$$
\mathcal{B}_{3}=\sqrt{-f} \nabla[f]_{\nu}\left(f^{\mu \nu} \delta \Gamma_{\mu \sigma}^{\sigma}[f]-f^{\mu \alpha} \delta \Gamma_{\mu \alpha}^{\nu}[f]\right) .
$$

\section{B Details of linearization}

In this appendix we present the detailed calculations of linearization of different terms in the action (1.4). To do so we will consider the following perturbations around an $\mathrm{AdS}_{3}$ solution

$$
g_{\mu \nu}=\bar{g}_{\mu \nu}+h_{\mu \nu}, \quad f_{\mu \nu}=\gamma\left(\bar{g}_{\mu \nu}+\rho_{\mu \nu}\right),
$$

where $\bar{g}_{\mu \nu}$ is the metric of the $A d S_{3}$ solution with the radius $\ell$. Assuming $g^{\mu \nu}$ and $f^{\mu \nu}$ as the inverse tensors corresponding to the metrics $g_{\mu \nu}$ and $f_{\mu \nu}$ respectively we have the contravariant version of eq. (B.1) as follows

$$
g^{\mu \nu}=\bar{g}^{\mu \nu}-h^{\mu \nu}+h_{\lambda}^{\mu} h^{\lambda \nu}, \quad f^{\mu \nu}=\gamma^{-1}\left(\bar{g}^{\mu \nu}-\rho^{\mu \nu}+\rho_{\lambda}^{\mu} \rho^{\lambda \nu}\right),
$$

where $h^{\mu \nu} \equiv \bar{g}^{\mu \alpha} \bar{g}^{\nu \beta} h_{\alpha \beta}$ and $\rho^{\mu \nu} \equiv \bar{g}^{\mu \alpha} \bar{g}^{\nu \beta} \rho_{\alpha \beta}$. The zeroth, first and second order of different terms of the action (1.4) with respect to the above perturbations are given by

$$
\begin{aligned}
& (\sqrt{-g} R[g])^{(0)}=\sqrt{-\bar{g}}\left(\frac{-6}{\ell^{2}}\right) \\
& (\sqrt{-g} R[g])^{(1)}=\sqrt{-\bar{g}}\left(-h_{; \mu}^{\mu}+h_{\mu \sigma}^{; \mu \sigma}-\frac{1}{\ell^{2}} h\right), \\
& (\sqrt{-g} R[g])^{(2)}=\sqrt{-\bar{g}}\left(-\frac{1}{4} h_{\nu \rho ; \mu} h^{\nu \rho ; \mu}+\frac{1}{2} h_{\mu \nu ; \rho} h^{\mu \rho ; \nu}-\frac{1}{2} h_{; \mu} h_{; \nu}^{\mu \nu}\right. \\
& \left.+\frac{1}{4} h^{; \mu} h_{; \mu}-\frac{1}{2 \ell^{2}}\left(h^{\mu \nu} h_{\mu \nu}-\frac{1}{2} h^{2}\right)\right) . \\
& (\sqrt{-f} R[f])^{(0)}=\gamma^{\frac{1}{2}} \sqrt{-\bar{g}}\left(\frac{-6}{\ell^{2}}\right), \\
& (\sqrt{-f} R[f])^{(1)}=\gamma^{-1} \sqrt{-\bar{g}}\left(-\rho_{; \mu}^{\mu}+\rho_{\mu \sigma}^{; \mu \sigma}-\frac{1}{\ell^{2}} \rho\right), \\
& (\sqrt{-f} R[f])^{(2)}=\gamma^{\frac{1}{2}} \sqrt{-\bar{g}}\left(-\frac{1}{4} \rho_{\nu \alpha ; \mu} \rho^{\nu \alpha ; \mu}+\frac{1}{2} \rho_{\mu \nu ; \alpha} \rho^{\mu \alpha ; \nu}-\frac{1}{2} \rho_{; \mu} \rho_{; \nu}^{\mu \nu}\right. \\
& \left.+\frac{1}{4} \rho^{; \mu} \rho_{; \mu}-\frac{1}{2 \ell^{2}}\left(\rho^{\mu \nu} \rho_{\mu \nu}-\frac{1}{2} \rho^{2}\right)\right) . \\
& \left(\sqrt{-g} f_{\mu \nu} G[g]^{\mu \nu}\right)^{(0)}=\gamma \sqrt{-\bar{g}}\left(\frac{3}{\ell^{2}}\right), \\
& \left(\sqrt{-g} f_{\mu \nu} G[g]^{\mu \nu}\right)^{(1)}=\gamma \sqrt{-\bar{g}}\left(\frac{1}{\ell^{2}}\left(\rho-\frac{1}{2} h\right)-\frac{1}{2}\left(-h_{; \mu}^{\mu}+h_{\mu \sigma}^{; \mu \sigma}\right)\right),
\end{aligned}
$$




$$
\begin{aligned}
& \left(\sqrt{-g} f_{\mu \nu} G[g]^{\mu \nu}\right)^{(2)}=\frac{\gamma}{2} \sqrt{-\bar{g}}\left(h_{; \nu}^{\nu \sigma} h_{\mu \sigma}^{; \mu}-h_{; \nu}^{\nu \sigma} h_{; \sigma}+h_{\nu}^{\sigma} h_{\mu \sigma ;}{ }^{\mu \nu}+\frac{1}{4} h^{; \mu} h_{; \mu}\right. \\
& +\frac{1}{2} h_{\mu \sigma ; \nu} h^{\mu \nu ; \sigma}-\frac{3}{4} h_{\mu \sigma ; \nu} h^{\mu \sigma ; \nu}-h^{\nu \sigma} h_{\rho \sigma ; \nu} \rho-\frac{1}{2} h h_{; \mu}^{\mu} \\
& +h\left(\frac{1}{2} h_{; \mu \nu}^{\mu \nu}+\frac{3}{4 \ell^{2}} h\right)+\rho^{\mu \nu}\left(-h_{\mu \nu ; \sigma}^{\sigma}-h_{; \mu \nu}+2 h_{\mu \sigma ; \nu}^{\sigma}\right) \\
& \left.+\rho\left(h_{; \mu}^{\mu}-h_{; \mu \nu}^{\mu \nu}-\frac{1}{\ell^{2}} h\right)+\frac{2}{\ell^{2}} h_{\mu \nu} \rho^{\mu \nu}-\frac{3}{2 \ell^{2}} h_{\mu \nu} h^{\mu \nu}\right) . \\
& \left(\sqrt{-g} \tilde{f}^{\mu \nu} f_{\mu \nu}\right)^{(0)}=3 \gamma^{2} \sqrt{-\bar{g}}, \quad\left(\sqrt{-g} \tilde{f}^{\mu \nu} f_{\mu \nu}\right)^{(1)}=\gamma^{2} \sqrt{-\bar{g}}\left(2 \rho-\frac{h}{2}\right), \\
& \left(\sqrt{-g} \tilde{f}^{\mu \nu} f_{\mu \nu}\right)^{(2)}=\gamma^{2} \sqrt{-\bar{g}}\left(\frac{9}{4} h^{\mu \nu} h_{\mu \nu}-4 h^{\mu \nu} \rho_{\mu \nu}+\rho^{\mu \nu} \rho_{\mu \nu}-\frac{5}{8} h^{2}+h \rho\right) . \\
& \left(\sqrt{-g} \tilde{f}^{2}\right)^{(0)}=9 \gamma^{2} \sqrt{-\bar{g}}, \quad\left(\sqrt{-g} \tilde{f}^{2}\right)^{(1)}=\gamma^{2} \sqrt{-\bar{g}}\left(6 \rho-\frac{3}{2} h\right), \\
& \left(\sqrt{-g} \tilde{f}^{2}\right)^{(2)}=\gamma^{2} \sqrt{-\bar{g}}\left(\rho h+\rho^{2}-\frac{7}{8} h^{2}+\frac{15}{4} h^{\mu \nu} h_{\mu \nu}-6 h^{\mu \nu} \rho_{\mu \nu}\right) . \\
& \left(\sqrt{-g} \Lambda_{g}\right)^{(0)}=\sqrt{-\bar{g}} \Lambda_{g}, \quad\left(\sqrt{-g} \Lambda_{g}\right)^{(1)}=\sqrt{-\bar{g}}\left(\frac{h}{2}\right) \Lambda_{g}, \\
& \left(\sqrt{-g} \Lambda_{g}\right)^{(2)}=\frac{1}{8} \sqrt{-\bar{g}}\left(h^{2}-2 h^{\mu \nu} h_{\mu \nu}\right) \Lambda_{g} . \\
& \left(\sqrt{-f} \Lambda_{f}\right)^{(0)}=\gamma^{\frac{3}{2}} \sqrt{-\bar{g}} \Lambda_{f}, \quad\left(\sqrt{-f} \Lambda_{f}\right)^{(1)}=\gamma^{\frac{3}{2}} \sqrt{-\bar{g}}\left(\frac{\rho}{2}\right) \Lambda_{f}, \\
& \left(\sqrt{-f} \Lambda_{f}\right)^{(2)}=\frac{1}{8} \gamma^{3 / 2} \sqrt{-\bar{g}}\left(\rho^{2}-2 \rho^{\mu \nu} \rho_{\mu \nu}\right) \Lambda_{f} .
\end{aligned}
$$

\section{Central charge from entropy function}

In this section we would like to provide another way to find the central charge (4.21) using Sen entropy function procedure [43]. In fact it was proved that for an extremal black hole whose near horizon geometry develops an $\mathrm{AdS}_{2}$ geometry the entropy can be found by minimizing the following entropy function

$$
S=2 \pi(e q-f)
$$

where $f=-2 \pi \int_{\text {horizon }} \mathcal{L}$, with $\mathcal{L}$ is Lagrangian density evaluated on the solution. The entropy is given by the minimal value of entropy function (see [43]). Here $e$ is a parameter of the solution whose conjugate is $q$ (see below for more details).

Let us now consider an extremal BTZ black hole in our model. The corresponding geometry can be expressed as $\mathrm{AdS}_{2}$ fibration of $\mathrm{AdS}_{3}$ whose metric may be parametrized as follows

$$
d s_{g}^{2}=v_{1}\left(-r^{2} d t^{2}+\frac{d r^{2}}{r^{2}}\right)+v_{2}(d \phi+e r d t)^{2}, \quad d s_{f}^{2}=\gamma d s_{g}^{2} .
$$

Of course for this metric to present an extremal BTZ black hole one should have $v_{2}=\frac{v_{1}}{e^{2}}$ and $v_{1}=\frac{\ell^{2}}{4}$. But for the moment we keep them two free independent parameters. These 
values should come automatically as one minimizes the entropy function. Note that the above metric is in the form of $A d S_{2} \times S^{1}$.

Now using the ansatz (C.2) and plugging it into the the Lagrangian density obtained from the action (1.4), one finds

$$
S=2 \pi e q+\frac{\pi}{G}\left(S_{G}+\kappa S_{\tilde{G}}\right),
$$

where

$$
\begin{aligned}
& S_{G}=\frac{e^{2} v_{2}^{\frac{3}{2}}}{16 v_{1}}(\gamma-2 \sigma)+\left(\left[\frac{3}{8} m^{2} \gamma^{2}+\frac{1}{2} \Lambda_{g}\right] v_{1}-\frac{1}{4}(\gamma-2 \sigma)\right) \sqrt{v_{2}}, \\
& S_{\tilde{G}}=-\frac{e^{2} v_{2}^{\frac{3}{2}}}{8 v_{1}} \sqrt{\gamma}+\frac{1}{2}\left(\gamma \Lambda_{f} v_{1}+1\right) \sqrt{\gamma v_{2}} .
\end{aligned}
$$

By extremizing the entropy function (C.3) with respect to $v_{1}, v_{2}$ and $e$, one gets

$$
\begin{aligned}
E^{v_{1}} & \equiv E_{G}^{v_{1}}+\kappa E_{\tilde{G}}^{v_{1}}=0, \\
E^{v_{2}} & \equiv E_{G}^{v_{2}}+\kappa E_{\tilde{G}}^{v_{2}}=0, \\
E^{e} & \equiv 2 \pi q+\frac{\pi}{G}\left(E_{G}^{e}+\kappa E_{\tilde{G}}^{e}\right)=0,
\end{aligned}
$$

where

$$
\begin{aligned}
& E_{G}^{v_{1}}=-\frac{e^{2} v_{2}^{\frac{3}{2}}}{16 v_{1}^{2}}(\gamma-2 \sigma)+\left(\frac{3}{8} m^{2} \gamma^{2}+\frac{1}{2} \Lambda_{g}\right) \sqrt{v_{2}}, \quad E_{\tilde{G}}^{v_{1}}=\frac{e^{2} v_{2}^{\frac{3}{2}}}{8 v_{1}^{2}} \sqrt{\gamma}+\frac{1}{2} \gamma^{\frac{3}{2}} \Lambda_{f} \sqrt{v_{2}}, \\
& E_{G}^{v_{2}}=\frac{3 e^{2} \sqrt{v_{2}}}{32 v_{1}}(\gamma-2 \sigma)+\left(\left[\frac{3}{16} m^{2} \gamma^{2}+\frac{1}{4} \Lambda_{g}\right] v_{1}-\frac{1}{8}(\gamma-2 \sigma)\right) \frac{1}{\sqrt{v_{2}}}, \\
& E_{\tilde{G}}^{v_{2}}=-\frac{3 e^{2} \sqrt{v_{2}}}{16 v_{1}} \sqrt{\gamma}+\frac{\sqrt{\gamma}}{4}\left(\gamma \Lambda_{f} v_{1}+1\right) \frac{1}{\sqrt{v_{2}}}, \quad E_{G}^{e}=\frac{e v_{2}^{\frac{3}{2}}}{8 v_{1}}(\gamma-2 \sigma), \quad E_{\tilde{G}}^{e}=-\frac{e v_{2}^{\frac{3}{2}}}{4 v_{1}} \sqrt{\gamma} .
\end{aligned}
$$

From the last equation in (C.5) we have

$$
q=\frac{\left(\sqrt{\gamma} \kappa+\sigma-\frac{\gamma}{2}\right) v_{2}^{\frac{3}{2}}}{8 G v_{1}} e
$$

while from the two other we arrive at

$$
E^{v_{1}}-2 \frac{v_{2}}{v_{1}} E^{v_{2}}=\frac{\pi \sqrt{v_{2}}}{2 G v_{1}^{2}}\left(\sqrt{\gamma} \kappa+\sigma-\frac{\gamma}{2}\right)\left(e^{2} v_{2}-v_{1}\right)=0
$$

Clearly $\sqrt{\gamma} \kappa+\sigma-\frac{\gamma}{2}$ can not be zero otherwise we get $q=0$ and a log gravity. Therefore the only solution of the above equation is

$$
v_{2}=\frac{v_{1}}{e^{2}}
$$

that is what was anticipated. One can then find a solution for $v_{1}$ by plugging the above expression of $v_{2}$ into the eq. (C.5)

$$
v_{1}=-\frac{\sqrt{\gamma} \kappa+\sigma-\frac{\gamma}{2}}{4 \kappa \gamma^{\frac{3}{2}} \Lambda_{f}+4 \Lambda_{g}+3 m^{2} \gamma^{2}} .
$$


On the other hand since the solution is locally AdS one has

$$
\Lambda_{g}=-\frac{1}{\ell^{2}}\left(\sigma+\frac{1}{4 m^{2} \ell^{2}}\right), \quad \Lambda_{f}=-m^{2}, \quad \gamma=\frac{1}{m^{2} \ell^{2}} .
$$

Note that using these values for the parameters of the model from (C.10) one gets $v_{1}=\frac{\ell^{2}}{4}$ as expected. Finally putting every things together and using the equations (C.7), (C.10) and (C.9) one arrivers at

$$
S=\frac{\pi \ell}{4 G e}\left(\sigma+\frac{\kappa}{m \ell}-\frac{1}{2 m^{2} \ell^{2}}\right)=2 \pi \sqrt{\frac{q \ell}{4 G}\left(\sigma-\frac{1}{2 m^{2} \ell^{2}}+\frac{\kappa}{m \ell}\right)} .
$$

which is the entropy of our extremal BTZ black hole that can be recast in to the following Cardy formula

$$
S=\frac{\pi^{2}}{3} T C
$$

where the central charge $C$ and Frolov-Thorne temperature $T$ are given by

$$
T=\frac{2}{\pi} \frac{\sqrt{G q}}{\sqrt{\ell\left(\sigma-\frac{1}{2 m^{2} \ell^{2}}+\frac{\kappa}{m \ell}\right)}}, \quad C=\frac{3 \ell}{2 G}\left(\sigma-\frac{1}{2 m^{2} \ell^{2}}+\frac{\kappa}{m \ell}\right),
$$

in agreement with the equation (4.21). The identification of the temperature may also be understood from the period of the compact direction in the ansatz (C.2) (see for example [44]).

\section{D $\tilde{\sigma}=-1$ case}

As we have already mentioned one could have considered either sign for the kinetic term of the metric $f_{\mu \nu}$. This option could be taken into account by multiplying the corresponding terms by $\tilde{\sigma}= \pm 1$ then the the action for metric $f_{\mu \nu}$ reads

$$
\frac{1}{16 \pi \tilde{G}} \int d^{3} x \sqrt{f}\left(\tilde{\sigma} R[f]-2 \Lambda_{f}\right) .
$$

The main parts of our paper have devoted to the case of $\tilde{\sigma}=1$. In this appendix we will briefly present the results of $\tilde{\sigma}=-1$ case. Indeed classical solutions we have found in section two can be mapped to this case by replacing $\kappa \rightarrow-\kappa$ and $\Lambda_{f} \rightarrow-\Lambda_{f}$.

Of course one needs to be more careful when we want to study the linearized action above a vacuum. Actually starting with the fluctuations (3.2) and going through all computations and using the same field redefinition as (3.7) one arrives at

$$
b=1, \quad a=\frac{m^{2} \ell^{2} \sigma+\frac{1}{2}}{m \ell \kappa+1} .
$$

Therefore, from the positivity of $\gamma$ we have $\sigma=-1$, in which the quadratic action reads

$$
S^{(2)}\left[h^{(0)}, h^{(m)}\right]=\frac{1}{16 \pi G} \int d^{3} x \sqrt{-\bar{g}}\left[\mathbb{A}_{0} h^{(0) \mu \nu}\left(\mathbb{G}^{\ell} h^{(0)}\right)_{\mu \nu}+\right.
$$




$$
\left.+\mathbb{A}_{m}\left\{h^{(m) \mu \nu}\left(\mathbb{G}^{\ell} h^{(m)}\right)_{\mu \nu}-\frac{1}{4} \mathbb{M}^{2}\left(h^{(m) \mu \nu} h_{\mu \nu}^{(m)}-\left(h^{(m)}\right)^{2}\right)\right\}\right]
$$

where

$$
\begin{aligned}
\mathbb{A}_{0} & =\sigma-\frac{1}{2 m^{2} \ell^{2}}-\frac{\kappa}{m \ell}, \\
\mathbb{A}_{m} & =\sigma+\left(\frac{3}{2}-2 a\right) \frac{1}{m^{2} \ell^{2}}-\frac{\kappa a^{2}}{m \ell} \\
\mathbb{M}^{2} & =-\frac{1}{\ell^{2}} \frac{(a-1)^{2}}{m^{2} \ell^{2} \mathbb{A}_{m}}
\end{aligned}
$$

One then should impose the following conditions to get a consistent theory

$$
\mathbb{A}_{0}>0, \quad \mathbb{A}_{m}>0, \quad \mathbb{M}^{2} \geq-\frac{1}{\ell^{2}}
$$

Using the same procedure as we have used in the body of paper we can find the central charge of the dual theory. Again in this case we get

$$
C=\frac{3 \ell}{2 G} \mathbb{A}_{0}
$$

It is evident from this expression that to get a consistent model $\sigma$ cannot be negative and therefore one should set $\sigma=1$. One then should look at the expressions (D.4) to see whether there is a range of parameters where both $\mathbb{A}_{0}$ and $\mathbb{A}_{m}$ are positive. Actually using e.g. Mathematica it is easy to see that in this case the parameters will not satisfy the conditions given in (D.5) and therefore for $\bar{\sigma}=-1$ one cannot get a consistent model.

Open Access. This article is distributed under the terms of the Creative Commons Attribution License (CC-BY 4.0), which permits any use, distribution and reproduction in any medium, provided the original author(s) and source are credited.

\section{References}

[1] E. Witten, Three-dimensional gravity revisited, arXiv:0706.3359 [INSPIRE].

[2] M. Bañados, C. Teitelboim and J. Zanelli, The black hole in three-dimensional space-time, Phys. Rev. Lett. 69 (1992) 1849 [hep-th/9204099] [INSPIRE].

[3] J.M. Maldacena, The large- $N$ limit of superconformal field theories and supergravity, Int. J. Theor. Phys. 38 (1999) 1113 [hep-th/9711200] [INSPIRE].

[4] Y.-F. Cai, F. Duplessis and E.N. Saridakis, $F(R)$ nonlinear massive theories of gravity and their cosmological implications, Phys. Rev. D 90 (2014) 064051 [arXiv:1307.7150] [INSPIRE].

[5] Y.-F. Cai and E.N. Saridakis, Cosmology of $F(R)$ nonlinear massive gravity, Phys. Rev. D 90 (2014) 063528 [arXiv: 1401.4418] [INSPIRE].

[6] S. Deser, R. Jackiw and S. Templeton, Topologically massive gauge theories, Annals Phys. 140 (1982) 372 [Erratum ibid. 185 (1988) 406] [Erratum ibid. 281 (2000) 409] [INSPIRE]. 
[7] E.A. Bergshoeff, O. Hohm and P.K. Townsend, Massive gravity in three dimensions, Phys. Rev. Lett. 102 (2009) 201301 [arXiv:0901.1766] [INSPIRE].

[8] M. Sadegh and A. Shirzad, Constraint strucrure of the three dimensional massive gravity, Phys. Rev. D 83 (2011) 084040 [arXiv:1010.2887] [INSPIRE].

[9] W. Li, W. Song and A. Strominger, Chiral gravity in three dimensions, JHEP 04 (2008) 082 [arXiv: 0801.4566] [INSPIRE].

[10] D. Grumiller and N. Johansson, Instability in cosmological topologically massive gravity at the chiral point, JHEP 07 (2008) 134 [arXiv:0805.2610] [INSPIRE].

[11] K. Skenderis, M. Taylor and B.C. van Rees, Topologically massive gravity and the AdS/CFT correspondence, JHEP 09 (2009) 045 [arXiv:0906.4926] [INSPIRE].

[12] D. Grumiller and I. Sachs, $A d S_{3} / L C F T_{2}$ - correlators in cosmological topologically massive gravity, JHEP 03 (2010) 012 [arXiv:0910.5241] [INSPIRE].

[13] D. Grumiller and O. Hohm, $A d S_{3} / L C F T_{2}$ : correlators in new massive gravity, Phys. Lett. B 686 (2010) 264 [arXiv:0911.4274] [INSPIRE].

[14] M. Alishahiha and A. Naseh, Holographic renormalization of new massive gravity, Phys. Rev. D 82 (2010) 104043 [arXiv: 1005.1544] [INSPIRE].

[15] M. Alishahiha and R. Fareghbal, D-dimensional log gravity, Phys. Rev. D 83 (2011) 084052 [arXiv: 1101.5891] [INSPIRE].

[16] I. Gullu, M. Gurses, T.C. Sisman and B. Tekin, AdS waves as exact solutions to quadratic gravity, Phys. Rev. D 83 (2011) 084015 [arXiv:1102.1921] [INSPIRE].

[17] E.A. Bergshoeff, O. Hohm, J. Rosseel and P.K. Townsend, Modes of log gravity, Phys. Rev. D 83 (2011) 104038 [arXiv:1102.4091] [INSPIRE].

[18] E. Bergshoeff, O. Hohm, W. Merbis, A.J. Routh and P.K. Townsend, Minimal massive 3D gravity, Class. Quant. Grav. 31 (2014) 145008 [arXiv: 1404.2867] [INSPIRE].

[19] O. Hohm, A. Routh, P.K. Townsend and B. Zhang, On the Hamiltonian form of $3 D$ massive gravity, Phys. Rev. D 86 (2012) 084035 [arXiv:1208.0038] [inSPIRE].

[20] C.J. Isham, A. Salam and J.A. Strathdee, F-dominance of gravity, Phys. Rev. D 3 (1971) 867 [INSPIRE].

[21] M. Bañados and S. Theisen, Three-dimensional massive gravity and the bigravity black hole, JHEP 11 (2009) 033 [arXiv:0909.1163] [InSPIRE].

[22] E.A. Bergshoeff, S. de Haan, O. Hohm, W. Merbis and P.K. Townsend, Zwei-dreibein gravity: a two-frame-field model of 3D massive gravity, Phys. Rev. Lett. 111 (2013) 111102 [Erratum ibid. 111 (2013) 259902] [arXiv:1307.2774] [INSPIRE].

[23] N. Arkani-Hamed, H. Georgi and M.D. Schwartz, Effective field theory for massive gravitons and gravity in theory space, Annals Phys. 305 (2003) 96 [hep-th/0210184] [INSPIRE].

[24] G. Dvali, O. Pujolàs and M. Redi, Non Pauli-Fierz massive gravitons, Phys. Rev. Lett. 101 (2008) 171303 [arXiv:0806.3762] [INSPIRE].

[25] A.R. Gover, A. Shaukat and A. Waldron, Tractors, mass and Weyl invariance, Nucl. Phys. B 812 (2009) 424 [arXiv:0810.2867] [INSPIRE].

[26] S. Carlip, S. Deser, A. Waldron and D.K. Wise, Cosmological topologically massive gravitons and photons, Class. Quant. Grav. 26 (2009) 075008 [arXiv:0803.3998] [INSPIRE]. 
[27] S. Carlip, S. Deser, A. Waldron and D.K. Wise, Topologically massive AdS gravity, Phys. Lett. B 666 (2008) 272 [arXiv:0807.0486] [INSPIRE].

[28] E.A. Bergshoeff, O. Hohm and P.K. Townsend, More on massive 3D gravity, Phys. Rev. D 79 (2009) 124042 [arXiv:0905.1259] [InSPIRE].

[29] S. Deser and A. Waldron, Partial masslessness of higher spins in (A)dS, Nucl. Phys. B 607 (2001) 577 [hep-th/0103198] [INSPIRE].

[30] S. Deser and A. Waldron, Gauge invariances and phases of massive higher spins in $(A) d S$, Phys. Rev. Lett. 87 (2001) 031601 [hep-th/0102166] [INSPIRE].

[31] S. Deser and A. Waldron, Null propagation of partially massless higher spins in (A)dS and cosmological constant speculations, Phys. Lett. B 513 (2001) 137 [hep-th/0105181] [INSPIRE].

[32] S. Deser and A. Waldron, Conformal invariance of partially massless higher spins, Phys. Lett. B 603 (2004) 30 [hep-th/0408155] [INSPIRE].

[33] B. Tekin, Partially massless spin-2 fields in string generated models, hep-th/0306178 [INSPIRE].

[34] M. Blagojevic and B. Cvetkovic, Extra gauge symmetries in BHT gravity, JHEP 03 (2011) 139 [arXiv:1103.2388] [INSPIRE].

[35] S. Alexandrov and C. Deffayet, On partially massless theory in 3 dimensions, JCAP 03 (2015) 043 [arXiv: 1410.2897] [INSPIRE].

[36] W. Merbis, Chern-Simons-like theories of gravity, arXiv:1411.6888 [INSPIRE].

[37] S.F. Hassan, A. Schmidt-May and M. von Strauss, On partially massless bimetric gravity, Phys. Lett. B 726 (2013) 834 [arXiv:1208.1797] [InSPIRE].

[38] S.F. Hassan, A. Schmidt-May and M. von Strauss, Bimetric theory and partial masslessness with Lanczos-Lovelock terms in arbitrary dimensions, Class. Quant. Grav. 30 (2013) 184010 [arXiv:1212.4525] [INSPIRE].

[39] J.L. Cardy, Operator content of two-dimensional conformally invariant theories, Nucl. Phys. B 270 (1986) 186 [INSPIRE].

[40] S. Carlip, Conformal field theory, $(2+1)$-dimensional gravity and the BTZ black hole, Class. Quant. Grav. 22 (2005) R85 [gr-qc/0503022] [INSPIRE].

[41] O. Hohm and E. Tonni, A boundary stress tensor for higher-derivative gravity in AdS and Lifshitz backgrounds, JHEP 04 (2010) 093 [arXiv:1001.3598] [INSPIRE].

[42] Y. Liu and Y.-W. Sun, Consistent boundary conditions for new massive gravity in $A d S_{3}$, JHEP 05 (2009) 039 [arXiv: 0903. 2933] [INSPIRE].

[43] A. Sen, Black hole entropy function and the attractor mechanism in higher derivative gravity, JHEP 09 (2005) 038 [hep-th/0506177] [INSPIRE].

[44] M. Guica, T. Hartman, W. Song and A. Strominger, The Kerr/CFT correspondence, Phys. Rev. D 80 (2009) 124008 [arXiv:0809.4266] [INSPIRE]. 